\title{
Editorial
}

\section{Do Physics Conferences Still Matter?}

In this issue, Silvan Schweber puts in historical context two notable events in twentieth-century physics, both held at Shelter Island. The first was the fabled Shelter Island conference of 1947, attended by most leading US physicists of the day, including Oppenheimer, Rabi, Feynman, Schwinger, and Bethe. These scientists, many of them fresh from the triumph of the Manhattan Project, were treated like royalty; several arrived by police escort arranged by a veteran of World War II, grateful to the men he thought had saved his life. Over the course of three days, these physicists learned of several startling new results, including the discovery of the Lamb shift, the measurement of the hyperfine structure of hydrogen and deuterium, and of results on meson absorption by the atmosphereall results that appeared to shake the foundations of the prevailing quantum theory. The conference inspired the physicists to go back and carefully reexamine that theory, resulting in modern quantum electrodynamics and the developments in quantum field theory that eventually led to the Standard Model.

The second Shelter Island conference, held in 1983, attempted to reunite as many as possible of the original participants. Many came, joined by leaders of the field who had emerged since the first conference. These participants were not received as heroes in the same way as their predecessors at the first conference. No shocking experimental results were announced. Schweber argues that the work of the participants did show that a powerful new synthesis of mathematics and physics now shaped theoretical physics. Still, the conference had a reflective and even retrospective air, marking, in effect, that the development of the Standard Model, begun at the first Shelter Island conference, was nearing completion. A fruitful and dramatic era in physics was drawing to a close.

Schweber's article asks us to consider the full, rich scene at that latter meeting, its larger historical and political context, and it poses the question of what it may portend for physics now. His article inspires us to ask: do physics conferences matter anymore?

Physics conferences today almost never attract all the leading figures in a field the way that the 1947 or even the 1983 conference did. Exciting results are communicated and digested much faster. Today's conference-goers hardly ever hear news they haven't already heard over the Internet, often via arXiv, which has practically supplanted traditional publishing in some areas of physics. What, then, is the purpose of such conferences, where most of the highly scheduled time is 
spent by speakers giving brief talks, cramming material into a dozen or so minutes, plus five minutes for questions? The biggest technological innovation introduced into conference going in the past century is PowerPoint, but it is not clear whether the long-term impact of this technology is to improve presentations or to deaden them. Given that all the material one hears at conferences could readily be presented online - and usually already is - what is the value of organizing a gathering of thousands of physicists? Some observers (such as the historian Sharon Traweek) point out how keenly physicists savor their "oral culture" on view at conferences, how much they value the social capital conferred by brilliant presentations or devastating criticisms. Participants often cite the benefits of the informal, unscheduled encounters in hallways or receptions. But is this worth the complexity and expense?

Humanists may be ahead of scientists technologically in this respect. They often pre-circulate conference papers so that participants can arrive prepared to ask carefully considered, thoughtful, and even provocative questions. Could such a system work for gatherings of physicists?

The change that Schweber's article describes between the first and second Shelter Island conferences makes us wonder whether physics conferences are in danger of becoming vestigial, ritualistic echoes of bygone days. Today they seem evidence of the relentless dampening impact of technology on human interactions, as well as the curious traditionalism of the practitioners of what one might have considered the most innovative science.

Physicists once pioneered open and provocative discussions as the way to advance their inquiries. What can be done to revitalize their conferences?

Robert P. Crease

Peter Pesic

The Editors Stony Brook, NY

USA 